\title{
Exercise-induced fatigue impairs visuomotor adaptability in physical education students
}

\author{
Zwierko T. ${ }^{1 \mathrm{ABCDE}}$, Wąsik J.2ABCDE \\ ${ }^{1}$ University of Szczecin, Institute of Physical Culture Sciences, Laboratory of Kinesiology in Functional and \\ Structural Human Research Center, Szczecin, Poland \\ ${ }^{2}$ Jan Dlugosz University in Czestochowa, Faculty of Health Sciences, Poland
}

Authors' Contribution: A - Study design; B - Data collection; C - Statistical analysis; D - Manuscript Preparation; E - Funds Collection.

\begin{abstract}
Purpose: $\quad$ Physical exercise has been shown to exert various effects on visuomotor processing and motor learning. The present study aimed to examine the impact of exercise with progressively increased physical load on consecutive stages of perceptual-motor learning. We compared the effectiveness of visuomotor adaptability in four subsequent trials during a complex coordination task performed in different conditions, including under conditions of progressively increased physical load, and in non-exercise resting control conditions.

Material: $\quad$ Twenty-seven physical education university students participated in this study. Participants were randomly assigned to one of two group: (1) an exercise experimental group $(n=14)$, or (2) a non-exercise resting control group $(n=13)$. Participants in the experimental group performed three 10-minute effort-tests with increasing intensity on a cycloergometer. Each participant was assigned individual workload values below the lactate threshold $\left(40 \% \mathrm{VO}_{2 \max }\right)$, at the lactate threshold $\left(60 \% \mathrm{VO}_{2 \max }\right)$, and above the lactate threshold $\left(80 \% \mathrm{VO}_{2 \max }\right)$. Four sessions of the two-hand coordination test included in the Vienna Test System were used to examine visuomotor adaptability variation. The total time duration, total error duration, and coordination difficulty were analyzed.

Results: $\quad$ There was a significant interaction between number of test repetitions and group (experimental, control) for total duration $\left(F_{(3,75)}=3.54, p=0.018\right)$. In particular, there was a significant reduction $(p=0.006)$ in duration in the control group after fourth test repetitions as compared to the baseline. In the experimental group, in contrast, there was a tendency for duration to increase after exercise above the lactate threshold intensity. There was also a significant interaction between test repetitions and group for total error duration $\left(F_{(3,75)}=\right.$ $3.14, p=0.03)$.

Conclusions: The results suggest that high intensity exercise can disrupt visuomotor processing during complex skill acquisition. These findings highlight the interplay between exercise intensity and motor control and learning, which in turn, has practical implications for developing and improving motor training and physical education programs.

Keywords: $\quad$ physical education students, motor control, learning, exercise.
\end{abstract}

\section{Introduction}

Physical education of students requires effectiveness methods of motor learning to the attainment of expertise in complex skills in different sports. Students need daily practice to acquire motor skills, coordinate large parts of muscles, and improve speed, accuracy, and coordination performance of fine, narrow movements that depend on visuomotor adaptation [1]. Visuomotor adaptation can be defined as the capacity to modify coordinated movements to adjust to changes in new environment conditions [2, 3]. Visuomotor adaptation is required for many basic tasks of daily living such as reaching and grasping objects, walking, cycling, car driving, and sports activity. To coordinate accurate and/or fast movements, the motor control system must adapt via dynamic changes in the musculoskeletal system and the configuration of body segments [4]. For effective visuomotor adaptability (VMA), several sensorimotor systems - including the visual system, central processing, and effector components - must function synergistically. In particular, integral components of hand-eye coordination task performance

(c) Zwierko T., Wąsik J., 2019

doi:10.15561/20755279.2019.0608 include projection of the visual field onto the retina, sensory transmission of information to the visual cortex, cognitive planning and motor programming, activation of arm muscles to initiate a particular action, focus of attention, and visual feedback [5].

It is generally assumed that visuomotor adaptation is the key factor that influences motor skill learning [6]. The level of an individual's motor learning improves with practice, which leads to relatively permanent changes in the acquisition of motor skill [7]. With practice, the control of movement execution becomes less dependent on cognitive processing and progressively switches to more automatic functioning [8].

Research studies have revealed different factors that can affect the effectiveness of perceptual-motor learning and performance, including task difficulty $[9,10]$, attention $[11,12]$, and memory abilities [13]. Moreover, stress and anxiety have been shown to decrease motor performance during the early stages of learning, but have no effect (or may even improve performance) during later learning stages [14, 15]. Furthermore, Hordacre et al. [16] reported perceptual-motor learning benefits during a pinch task following increased stress and anxiety induced by 
the mental arithmetic task. In particular, the investigators observed a reduction in both reaction time and movement time across the learning period. These findings suggest that levels of stress and anxiety are associated with adaptive changes in motor learning.

Physical exercise has been reported to exert various effects on visuomotor processing and motor learning [17-20]. On one hand, researchers have concluded that exercise has a beneficial effect on the excitation and activity of the peripheral and central nervous systems [21, 22]. On the other hand, other groups have reported that intense physical activity may interfere with neural signal transmission [23-25]. The mechanism of exercise-induced effects on visuomotor processing remains unclear [26].

The purpose of motor learning is to produce more effective movements [3]. The present study aimed to systematically investigate the effects of exercise on VMA in physical education students. Thus, we examined the effect of exercise with progressively increased physical load on consecutive stages of perceptual-motor learning. We compared the effectiveness of VMA in four subsequent trials during a complex coordination task performed in different conditions, including under conditions of progressively increased physical load, and in non-exercise resting control conditions.

\section{Material and methods}

Participants.

Twenty-seven physical education students from University of Szczecin, Poland ( $M \pm S D$ age: $20.19 \pm$ 1.4 years) participated in his study. Participants were randomly assigned to one of two group: (1) an exercise experimental group $(\mathrm{n}=14)$, and (2) a non-exercise resting control group $(\mathrm{n}=13)$. The local Bioethical Committee approved the research project.

\section{Research Design.}

\section{Preliminary protocol}

Participants in the experimental group completed an effort test that involved an incremental increase in intensity using a cycloergometer (Monark E834, Varberg, Sweden). The experiment began with a 10-min rest period in a reclined position. After $10 \mathrm{~min}$, a blood sample was collected from a finger for biochemical determinations. Participants then completed a 5-min warm-up period at 25 watts (W). The effort-test commenced at $70 \mathrm{~W}$, with 70 revolutions per min (rpm). The exercise continued with an increasing workload $(20 \mathrm{~W}$ increments every 3 min) until refusal. During the last $15 \mathrm{sec}$ of each 3-min effort at a given workload, capillary blood samples were drawn from a fingertip for enzymatic determination of blood lactate concentration (Dr. Lange Cuvette Test LKM 140, Germany). Lactate concentration was determined using miniphotometer LP 20 Plus (Dr Lange, Germany). Resting heart rate and change in heart rate during exercise were measured using a Polar S610 heart rate monitor (Polar, Finland). Oxygen consumption during exercise was estimated using an Oxycon gas analyzer (Jaeger, Germany). Individual lactate threshold was calculated using a linear regression graph log lactate and the log of effort intensity. Based on the results of the exercise test, each subject was assigned an individual workload value (W) at various levels: (1) a $40 \% \mathrm{VO}_{2 \max }$ (i.e., load value below the lactate threshold), (2) a lactate threshold range, which in the case of all participants was between $65-75 \% \mathrm{VO}_{2 \max }$, and (3) an $80 \% \mathrm{VO}_{2 \max }$ (i.e., above lactate threshold).

The two-hand coordination test (i.e., the VMA test) included in the Vienna Test System (Schuhfried, Austria) was used to examine VMA. During the VMA test, participants used two control elements (i.e., joysticks) to move a cursor along a track shown on a monitor (see Figure 1). The cursor can be moved horizontally using one joystick, and moved vertically using the other joystick. Participants were instructed to run the track through from start to finish as quickly as possible. An error is counted each time the cursor goes off the track. The track consists of three sections of varying difficulty (circular arc, $\mathrm{V}$-shape, inverted L). Speed and accuracy of the run on each track was scored, including the following variables: total mean duration $[\mathrm{s}]$, total mean error duration [s], and coordination difficulty [s] (i.e., time difference standardized to the length of the path between sections with or without need for coordination).

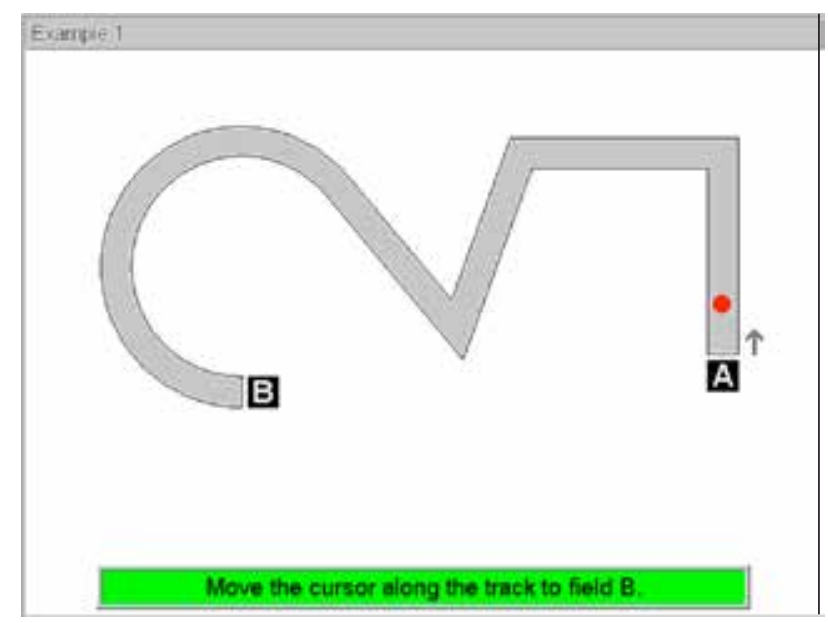

Figure 1. Scheme of the tract in the two-hand coordination test (VMA test).

\section{Procedure}

The experiment was carried out 5 days after the efforttest that determined the maximal oxygen uptake (i.e., $\mathrm{VO}_{2} \max$ ). The first VMA test (T1) was performed at rest. Next, all participants completed a 5-min warm-up on the cycloergometer $(25 \mathrm{~W})$. Participants then completed a 10-min effort at intensity below lactate threshold (i.e., $40 \% \mathrm{VO}_{2 \max }$ ). The second VMA test (T2) was performed immediately after the effort test. Participants then performed a 10-min effort at lactate threshold (i.e., $60 \% \mathrm{VO}_{2 \max }$ ) and after that, the third VMA test (T3) was conducted. Next, participants performed a 10-min effort at intensity above the lactate threshold (i.e., $80 \% \mathrm{VO}_{2 \max }$ ), which was followed immediately by the VMT test (T4). During cycling, participants maintained a constant 
frequency of revolutions (68-72 rate per min). Heart rate was monitored throughout the experiment. For the nonexercise resting control group, four VMA test (T1-T4) were conducted, with a 10-min break between each test.

Statistical methods

All data are expressed as mean $(M) \pm$ standard deviation $(S D)$. The assumption of normality was tested using the Shapiro-Wilk test. The dependent measures (i.e., total mean duration, total error duration, coordination difficulty) were analyzed separately using a two-way repeated measures analysis of variance (ANOVA) to test for significant effects of the between-subjects factor (group: experimental, control) and the within-subjects factor (test repetitions: T1, T2, T3, T4). Post hoc tests were performed, results were considered significant at $p$ $<0.05$, Bonferroni corrected.

\section{Results}

Descriptive statistics of the VMA test parameters for the experimental and control groups are presented in Table 1.

Effects on total mean duration of visuomotor adaptability test

The analyses of the total mean duration of VMA test revealed no significant main effect of group $\left(F_{(1,55)}=\right.$ $\left.0.01, p=0.938, \eta p^{2}=0.001\right)$, a significant main effect of test repetitions $\left(F_{(3,75)}=5.14, p=0.003, \eta p^{2}=0.17\right)$, and a significant group $\mathrm{x}$ test repetitions interaction $\left(F_{(3,75)}=3.54, p=0.018, \eta p^{2}=0.12\right)$ (Figure 1). The test repetitions factor significantly differentiated the plot of the total mean duration changes in the control group (i.e., VMA test trials at rest) as compared to the experimental group (i.e., VMA test trials in progressively increase physical effort). In the control group, the total duration of the fourth VMA test (T4) was shorter than in the initial measurement (T1) (delta: $2.275 \mathrm{~s}, p=0.006$ ). In contrast, there were no significant changes $(p>0.05)$ in subsequent VMA test trials in the experimental group.

There was a significant reduction ( $\mathrm{T} 1 \mathrm{vs} . \mathrm{T} 4, p=0.006$ ) in total duration in the control group (denoted with *). In contrast, in the experimental group, the total duration did not significantly change during VMA test repetitions in exercise conditions.

Effects on total error duration of visuomotor adaptability test

The total error duration did not significantly differ between groups $\left(F_{(1,25)}=0.39 .71, p=0.537, \eta p^{2}=0.02\right)$, or vary by test repetitions $\left(F_{(3,75)}=1.90, p=0.136, \eta p^{2}\right.$ $=0.07)$. However, there was a significant interaction between group and test repetitions $\left(F_{(3,75)}=3.14, p=0.03\right.$, $\eta p^{2}=0.11$ ) for total error duration (Figure 2). Indeed, in the experimental group there was a tendency for total error duration to decrease after the first effort (T1), and increase after the second (T2) and third efforts (T3). In the control group, in contrast, total error duration gradually decreased over time.

Effects on coordination difficulty of visuomotor adaptability test

Types of variances in the coordination difficulty did not differ between the experimental and control groups

Table 1. Mean, standard deviation (SD) of VMA test parameters in the experimental and control groups.

$M \pm S D$

VMA test parameters

Experimental group

Total mean duration $[\mathrm{s}]$

T1

T2

T3

T4

Total error duration [s]

T1

T2

T3

T4

Coordination difficulty [s]

T1

$\mathrm{T} 2$

T3

T4
$16.58 \pm 3.43$

$15.35 \pm 3.63$

$15.13 \pm 3.07$

$16.32 \pm 4.11$

$0.67 \pm 0.42$

$0.47 \pm 0.37$

$0.48 \pm 0.23$

$0.78 \pm 0.32$

$2.02 \pm 0.69$

$1.94 \pm 0.65$

$1.91 \pm 0.47$

$2.00 \pm 0.33$
$2.24 \pm 0.64$

\section{Control group}

$16.90 \pm 4.18$

$15.99 \pm 3.08$

$15.47 \pm 2.95$

$14.63 \pm 3.12$

$0.65 \pm 0.50$

$0.53 \pm 0.40$

$0.47 \pm 0.29$

$0.37 \pm 0.22$

$2.10 \pm 0.48$

$2.00 \pm 0.57$

$1.88 \pm 0.48$ 


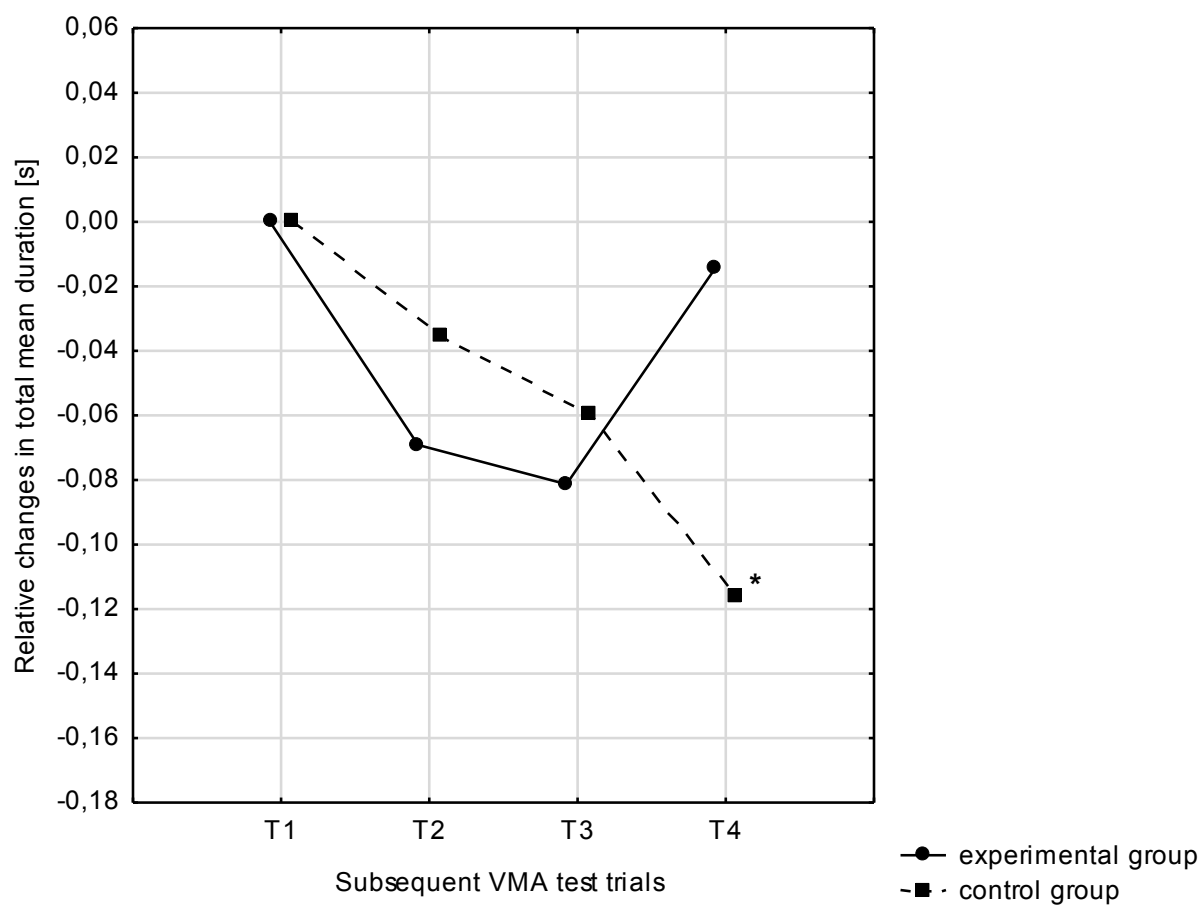

Figure 2. Interaction between group and test repetitions for the total duration $\left(F_{(3,75)}=3.54, p=0.018, \eta p^{2}=0.12\right)$.

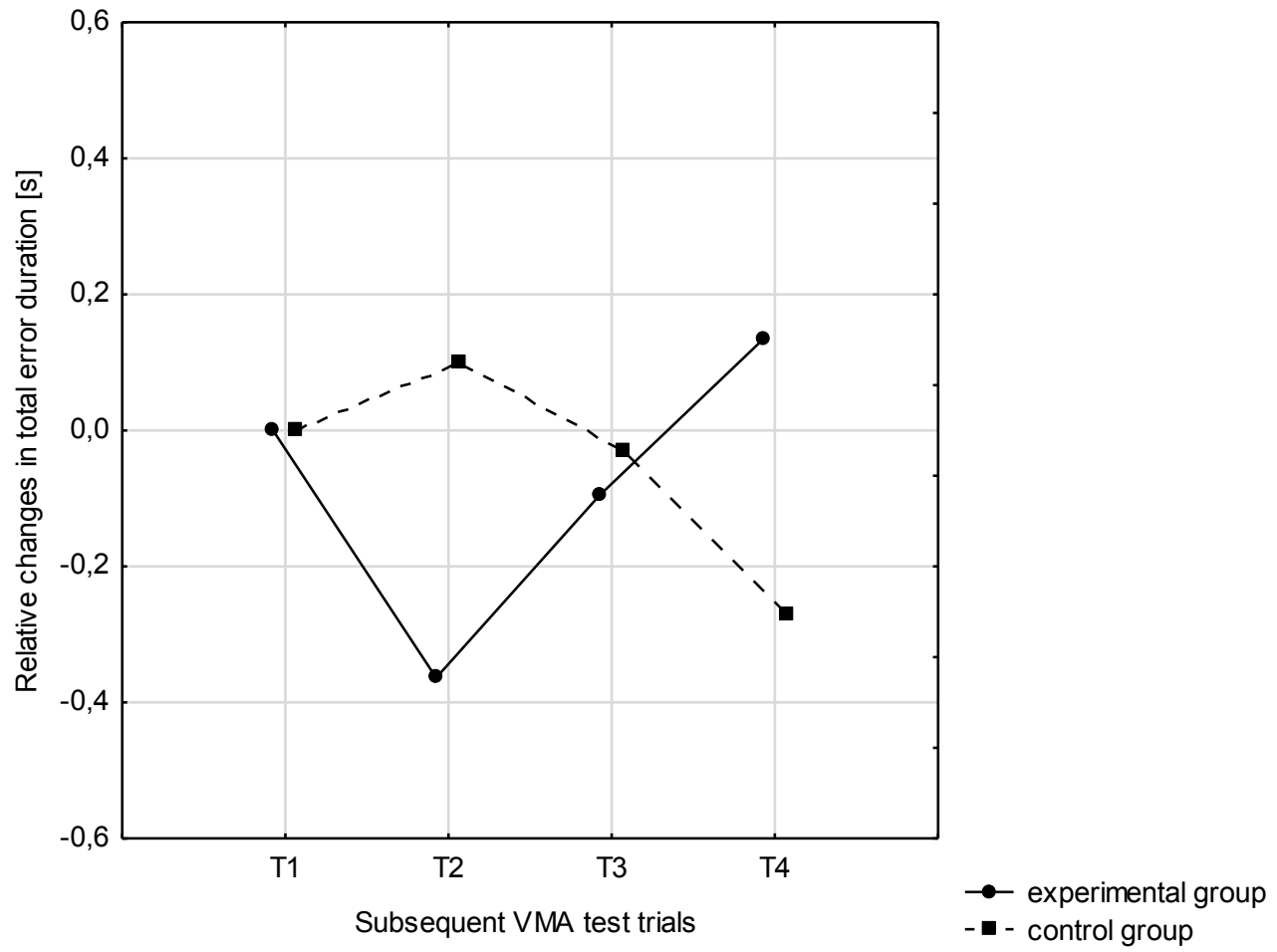

Figure 3. Interaction plot of relative changes of test repetitions and group for the total error duration $\left(F_{(3,75)}=3.14, p\right.$ $=0.03, n p^{2}=0.11$ ).

$\left(F_{(1,25)}=0.22, p=0.641, \eta p^{2}=0.01\right)$. The plot of changes in coordination difficulty parameter over consecutive repetitions did not differentiate the experimental from the control group $\left(F_{(3,75)}=1.85, p=0.145, \eta p^{2}=0.07\right)$. Moreover, there was no significant interaction between group and test repetitions $\left(F_{(3,75)}=1.33, p=0.270, \eta p^{2}=\right.$ $0.05)$.

\section{Discussion}

The main finding in our study was that exercise did not improve VMA, as compared to motor practice alone. Increased physical effort did not cause significant changes in the speed and accuracy of VMA in the experimental group. We observed only a tendency for the direction and size of changes that depended on the intensity 
of the physical effort. Specifically, as the intensity of physical effort increased, the speed and accuracy of VMA improved until a critical value of exercise intensity was reached. Following the critical value, speed and accuracy parameters worsened. However, the results of the experimental versus control group showed significantly different plots in terms of variation in the recorded parameters. Indeed, total test duration was significantly better after four repetitions in the control group. This was in contrast to the experimental group, which tended to show a worsening of parameters after four repetitions (Figure 2). A similar tendency was observed for total error duration (Figure 3).

Exercise has an influence on human visuomotor processing and results of several studies indicate that the motor learning process can be facilitated. For instance, Statton et al. [20] investigated the effects of acute bouts of moderate intensity aerobic exercise on the acquisition and retention of a new motor skill (i.e., a sequential visual isometric pinch task). The investigators found that aerobic exercise led to significantly greater performance on the motor skill learning, but no effects on results of a retention test. Perini et al. [22] demonstrated that aerobic exercise can enhance the ability of young adults male to acquire skills during an orientation discrimination task that involved primary visual cortex activity, and also during a simple thumb abduction motor task that relies on primary motor cortex activity. In light of these results, the authors suggested that moderate intensity exercise can enhance brain plasticity. There is also evidence that brain-derived neurotrophic factor (BDNF) moderates the effects of physical activity on the resultant cognitive and neuroplastic changes [26]. Furthermore, various experimental studies have concluded that acute and long-term participation in moderate-intensity exercise can enhance working memory, short- and long-term memory, and executive function [18, 19, 27], which can promote motor learning effects. It is generally confirmed that exercise at higher intensities results in greater elevations in catecholamines, which cause significant effects on attentional, perceptual, and working memory processing by increasing neural blood-glucose levels [28]. Specifically, enhances were noted in noradrenaline-modulated cortical processing, which has a crucial influence on physiological arousal, cognition, and attention [16, 29, 30].

Relevant to our results, several experimental studies applied visuomotor tasks that require tracking patterns presented on a screen and did not find improved learning and retention of motor accuracy after moderate and intensity exercise. For example, in Snow et al. [31], participants completed a procedure that included aerobic exercise followed immediately by practice with a novel tracking motor task. Exercise consisted of 30 minutes of continuous cycling at $60 \%$ peak $\mathrm{O} 2$ uptake. There were no differences in visuomotor skill acquisition between rest and after exercise $(p=0.066)$, and for retention test
( $p=0.761)$ that occurred 24 hours after the intervention. Similarly, Singh et al. [32] used a moderate-intensity exercise protocol to assess response time during a bimanual task and did not obtain any significant differences between the exercise and control groups. A recent study by Stranda et al. [33] found no effects of moderate-intensity aerobic exercise (i.e., $65 \%$ HRmax) conducted before each practice trial (3x/week for 4 weeks) on speed and accuracy parameters in a novel keyboard typing task. Similar to our procedure, the experiments include both an exercise and a non-exercise resting control group. All participants in the Standa et al. [33] study showed an improvement in both speed and accuracy during the keyboard typing task. However, the range of improvement on both speed and accuracy task parameters did not significantly differ between the exercise and control groups in the retention test.

In our opinion, it is possible that intensity is a key modulator of the effects of exercise on changes in complex VMA. Moreover, we argue that the learning of more complex coordination tasks are not facilitated by high intensity exercise-induced fatigue [28]. For example, $\mathrm{Hu}$ et al. [34] investigated the effects of muscle fatigue (i.e., continuous submaximal pinch strength) on coordination of force directions during precision grip. Muscle fatigue interfered with grasping stability by decreasing the average coordination angle across the thumb and index finger, and by reducing the projection angle of the index finger in the ipsilateral hand. Moreover, fatigue may influence sensorimotor integration, thereby causing a reduction in movement precision [35]. Due to fatigue, the central nervous system may not adapt to the altered relation between neural output and sensory feedback. In our study, this lack of adaptation may have led to the observed variation in the experimental and control groups during the T4 task, wherein the experimental group performed effort above lactate threshold (i.e., $80 \% \dot{\mathrm{V}}$ $\left.\mathrm{O}_{2 \max }\right)$. Previous results from electrophysiology studies indicate that exercise-induced fatigue can affect different stages of information processing, i.e. sensory, central, and effector $[25,36,37]$.

\section{Conclusions}

Results of the present study suggest that high intensity exercise can disrupt visuomotor processing during complex skill acquisition. This observation has important implications for the methodological approach of a physical education study program. These findings highlight the interplay between exercise intensity and motor control and learning, which in turn, has practical implications for developing and improving motor training and physical education programs.

\section{Conflicts of Interest}

The authors declare no conflict of interest. 


\section{References}

1. Altaee HA, Alghurery WT, Abdulqader HD. Effect of the Six Thinking Hats Exercises on Situational Embarrassment and Performing some of Skills on Vaulting Table in Gymnastics. International Journal of Advanced Sport Sciences Research. 2014;2(4):365-72.

2. Doyon J, Penhune V, Ungerleider LG. Distinct contribution of the cortico-striatal and cortico-cerebellar systems to motor skill learning. Neuropsychologia. 2003;41(3):252-62. https://doi.org/10.1016/S0028-3932(02)00158-6

3. Krakauer JW, Hadjiosif AM, Xu J, Wong AL, Haith AM. Motor Learning. Compr Physiol. 2019;9(2):613-63. https://doi.org/10.1002/cphy.c170043

4. Shadmehr R, Smith MA, Krakauer JW. Error correction, sensory prediction, and adaptation in motor control. Annu Rev Neurosci. 2010;33:89-108. https://doi.org/10.1146/annurev-neuro-060909-153135

5. Bard C, Fleuy M, Hay L. Development of eye hand coordination across the life span. Columbia: SC University of South Carolina Press; 1990.

6. SeidlerRD. Differential effects of age on sequence learning and sensorimotoradaptation. Brain Res Bull. 2006;70(4-6):337-46. https://doi.org/10.1016/j.brainresbull.2006.06.008

7. Schmidt RA, Wrisberg CA. Motor learning and performance. A problem - based learning approach. Champaingn Human Kinetics Books; 2000.

8. Koedijker JM, Poolton JM, Maxwell JP, Oudejans RR, Beek PJ, Masters RS. Attention and time constraints in perceptualmotor learning and performance: instruction, analogy, and skill level. Conscious Cogn. 2011;20(2):245-56. https://doi.org/10.1016/j.concog.2010.08.002

9. Andrieux M, Boutin A, Thon B. Self-Control of Task Difficulty During Early Practice Promotes Motor Skill Learning. J Mot Behav. 2016;48(1):57-65. https://doi.org/10.1080/00222895.2015.1037879

10.Andrieux M, Danna J, Thon B. Self-control of task difficulty during training enhances motor learning of a complex coincidence-anticipation task. Res $Q$ Exerc Sport. 2012;83(1):27-35. https://doi.org/10.1080/02701367.2012.10599822

11.Castaneda B, Gray R. Effects of focus of attention on baseball batting performance in players of differing skill levels. J Sport Exerc Psychol. 2007;29(1):60-77. https://doi.org/10.1123/jsep.29.1.60

12.Song JH. The role of attention in motor control and learning. Curr Opin Psychol. 2019;29:261-5. https://doi.org/10.1016/j.copsyc.2019.08.002

13.Beilock SL, Wierenga SA, Carr TH. Expertise, attention, and memory in sensorimotor skill execution: impact of novel task constraints on dual-task performance and episodic memory. $Q J$ Exp Psychol A. 2002;55(4):1211-40. https://doi.org/10.1080/02724980244000170

14. Vine SJ, Freeman P, Moore LJ, Chandra-Ramanan R, Wilson MR. Evaluating stress as a challenge is associated with superior attentional control and motor skill performance: testing the predictions of the biopsychosocial model of challenge and threat. J Exp Psychol Appl. 2013;19(3):185-94. https://doi.org/10.1037/a0034106

15. Vit M, Houdek M, Sebera M. Reaction time and stress tolerance of policeofficersinspecificandnon-specifictestsinprofessional self-defence training. Phys Activ Rev. 2019; 7: 193-200. https://doi.org/10.16926/par.2019.07.23

16.Hordacre B, Immink MA, Ridding MC, Hillier S. Perceptual-motor learning benefits from increased stress and anxiety. Hum Mov Sci. 2016;49:36-46. https://doi.org/10.1016/j.humov.2016.06.002

17.Audiffren M, Tomporowski PD, Zagrodnik J. Acute aerobic exercise and information processing: modulation of executive control in a Random Number Generation task. Acta Psychol (Amst). 2009;132(1):85-95. https://doi.org/10.1016/j.actpsy.2009.06.008

18.Lambourne K, Tomporowski P. The effect of exerciseinduced arousal on cognitive task performance: a meta-regression analysis. Brain Res. 2010;1341:12-24. https://doi.org/10.1016/j.brainres.2010.03.091

19.McMorris T, Sproule J, Turner A, Hale BJ. Acute, intermediate intensity exercise, and speed and accuracy in working memory tasks: a meta-analytical comparison of effects. Physiol Behav. 2011;102(3-4):421-8. https://doi. org/10.1016/j.physbeh.2010.12.007

20.Statton MA, Encarnacion M, Celnik P, Bastian AJ. A Single Bout of Moderate Aerobic Exercise Improves Motor Skill Acquisition. PLoS One. 2015;10(10):e0141393. https://doi.org/10.1371/journal.pone.0141393

21.Davranche K, Burle B, Audiffren M, Hasbroucq T. Physical exercise facilitates motor processes in simple reaction time performance: an electromyographic analysis. Neurosci Lett. 2006;396(1):54-6. https://doi.org/10.1016/j.neulet.2005.11.008

22.Perini R, Bortoletto M, Capogrosso M, Fertonani A, Miniussi C. Acute effects of aerobic exercise promote learning. Sci Rep. 2016;6:25440. https://doi.org/10.1038/srep25440

23.Ando S, Kimura T, Hamada T, Kokubu M, Moritani $\mathrm{T}$, Oda $\mathrm{S}$. Increase in reaction time for the peripheral visual field during exercise above the ventilatory threshold. Eur J Appl Physiol. 2005;94(4):461-7. https://doi.org/10.1007/s00421-005-1330-7

24.Zwierko T, Czepita D, Lubinski W. The effect of physical effort on retinal activity in the human eye: rod and cone flicker electroretinogram studies. Graefes Arch Clin Exp Ophthalmol. 2010;248(5):659-66. https://doi.org/10.1007/s00417-010-1305-1

25.Tomporowski PD. Effects of acute bouts of exercise on cognition. Acta Psychol (Amst). 2003;112(3):297-324. https://doi.org/10.1016/s0001-6918(02)00134-8

26.Huang T, Larsen KT, Ried-Larsen M, Moller NC, Andersen LB. The effects of physical activity and exercise on brain-derived neurotrophic factor in healthy humans: A review. Scand J Med Sci Sports. 2014;24(1):1-10. https://doi.org/10.1111/sms.12069

27.Roig M, Nordbrandt S, Geertsen SS, Nielsen JB. The effects of cardiovascular exercise on human memory: a review with meta-analysis. Neurosci Biobehav Rev. 2013;37(8):1645-66. https://doi.org/10.1016/j.neubiorev.2013.06.012

28.McMorris T,HaleBJ.Differential effects ofdifferingintensities of acute exercise on speed and accuracy of cognition: a metaanalytical investigation. Brain Cogn. 2012;80(3):338-51. https://doi.org/10.1016/j.bandc.2012.09.001

29.McDonnell MN, Smith AE, Mackintosh SF. Aerobic exercise to improve cognitive function in adults with neurological disorders: a systematic review. Arch Phys Med Rehabil. 2011;92(7):1044-52. https://doi.org/10.1016/j.apmr.2011.01.021

30.McMorris T. Developing the catecholamines hypothesis for the acute exercise-cognition interaction in humans: Lessons from animal studies. Physiol Behav. 2016;165:291-9. https://doi.org/10.1016/j.physbeh.2016.08.011

31.Snow NJ, Mang CS, Roig M, McDonnell MN, Campbell KL, Boyd LA. The Effect of an Acute Bout of Moderate- 
Intensity Aerobic Exercise on Motor Learning of a Continuous Tracking Task. PLoS One. 2016;11(2):e0150039. https://doi.org/10.1371/journal.pone.0150039

32.Singh AM, Neva JL, Staines WR. Aerobic exercise enhances neural correlates of motor skill learning. Behav Brain Res. 2016;301:19-26. https://doi.org/10.1016/j.bbr.2015.12.020

33. Stranda $H$, Haga $M$, Sigmundsson $H$, Loras $H$. The Effect of Aerobic Exercise on Speed and Accuracy Task Components in Motor Learning. Sports (Basel). 2019;7(3). https://doi.org/10.3390/sports7030054

34.Hu W, Wei N, Li ZM, Li K. Effects of muscle fatigue on directional coordination of fingertip forces during precision grip. PLoS One. 2018;13(12):e0208740. https://doi.org/10.1371/journal.pone.0208740

35. Todd G, Gandevia SC, Taylor JL. Change in manipulation with muscle fatigue. Eur J Neurosci. 2010;32(10):1686-94. https://doi.org/10.1111/j.1460-9568.2010.07444.x

36.Zwierko T, Lubinski W, Lubkowska A, Niechwiej-Szwedo E, Czepita D. The effect of progressively increased physical efforts on visual evoked potentials in volleyball players and non-athletes. $J$ Sports Sci. 2011;29(14):1563-72. https://doi.org/10.1080/02640414.2011.605166

37.Liu SH, Lin CB, Chen Y, Chen W, Huang TS, Hsu CY. An EMG Patch for the Real-Time Monitoring of Muscle-Fatigue Conditions During Exercise. Sensors (Basel). 2019;19(14). https://doi.org/10.3390/s19143108

\section{Information about the authors:}

Zwierko T. ' http://orcid.org/0000-0003-0519-9461; teresa.zwierko@usz.edu.pl; Institute of Physical Culture Sciences, Laboratory of Kinesiology in Functional and Structural Human Research Center, University of Szczecin; Aleja Papieża Jana Pawła II 22A, 70-453, Szczecin, Poland.

Wąsik J.; (Corresponding Author); http://orcid.org/0000-0002-6285-7283; j.wasik@ujd.edu.pl; Institute of Physical Education, Tourism and Physiotherapy, Jan Długosz University in Czestochowa; ul. Waszyngtona 4/8, 42-200 Częstochowa, Poland.

\section{Cite this article as:}

Zwierko T, Wąsik J. Exercise-induced fatigue impairs visuomotor adaptability in physical education students. Physical education of students, 2019;23(6):327-333.

https://doi.org/10.15561/20755279.2019.0608

This is an Open Access article distributed under the terms of the Creative Commons Attribution License, which permits unrestricted use, distribution, and reproduction in any medium, provided the original work is properly cited http://creativecommons.org/licenses/by/4.0/deed.en

Received: 30.09 .2019

Accepted: 29.10.2019; Published: 18.12.2019 